\title{
Ontogeny of persistence: Immediate extinction effects in preweanling and weanling rats
}

\author{
DAVID R. BURDETTE, STEPHEN BRAKE, JAW-SY CHEN, and ABRAM AMSEL \\ The University of Texas at Austin, Austin, Texas 78712
}

\begin{abstract}
In Experiment I rats were trained for $2^{1 / 2}$ days under partial (PRF) or continuous reinforcement (CRF) conditions starting at $18,22,28$, or 36 days of age and were then subjected to immediate extinction. At all ages there was a strong partial reinforcement extinction effect (PREE), and absolute size of PREE was greatest in the youngest rats. Rate of extinction increased as a function of age following both CRF and PRF. In Experiment II the youngest and oldest age groups of Experiment I were run under the two reward conditions of Experiment I and in a third condition, PRF with number of rewards rather than trials equated to CRF (PRF-R). The PRF-R and PRF groups were not different in extinction, and both were more persistent than CRF. The youngest rats were again more persistent than the oldest, particularly after PRF training. In Experiment III it was shown that the well-known paradoxical effect, greater reward in CRF acquisition leads to faster extinction, operates in our youngest and oldest animals, but is more pronounced in the oldest. The results are discussed in terms of whether they require different explanations than those often applied to extinction data from adult rats.
\end{abstract}

We seem to know much more about the ontogeny of aversive (fear) conditioning in rats than about appetitive learning, perhaps because it seems easier to equate motivational factors at different ages in aversive than in appetitive learning (Campbell, 1967). However, we do have a little information on how level of maturity in rats affects learning in appetitive situations. Weanling rats are reported to be inferior to adults in learning a barpress response for food reward and in forming a lightdark discrimination (Campbell, Jaynes, \& Misanin, 1968). Similarly, juvenile rats (less than 50 days of age) do less well on a spatial discrimination than adults (Bronstein \& Spear, 1972). The younger rats in the studies of Campbell and his colleagues also show poorer retention of both a learned barpress and light-dark discrimination (Campbell et al., 1968), unless there are periodic reexposures of the rats to the experimental task over the retention interval (Campbell \& Jaynes, 1969).

While these experiments tell us something about the ontogeny of appetitive learning, they fall short of being decisive in several respects. First, the number of ages investigated is small over the range of ages investigated. This makes it difficult to determine the age at which an adult pattern first appears, and says little about the abruptness of the transition. A second related point is that, when training sessions are conducted over an extended period of time, it is difficult to separate changes in performance related to developmental factors from changes resulting from amount or duration of training. Finally, experimental treatment of the young. est subjects in these studies on appetitive learning usually begins in the fourth postnatal week or later,

This research was supported by Grant BMS 74-19696 from the National Science Foundation. The authors acknowledge the assistance of Sandra Nelson, Cathy Radek, and Lindsay Rosin in various phases of this research. Reprint requests should be in various phases of this research. Reprint requests should be
addressed to A. Amsel, Department of Psychology, University of Texas at Austin, Austin, Texas 78712 . which means a critical phase of development occurring in the rat at about 20-25 days of age, with regard to physiology (Altman \& Das, 1965; Campbell, Lytle, \& Fibiger, 1969) and behavior (Bronstein, Neiman, Wolkoff, \& Levine, 1974; Egger, Livesey, \& Dawson, 1973), is not studied at all.

Systematic experimental study of the ontogeny of learning offers benefits much like those derived from the study of phyletic differences (Bitterman, 1965). There is first of all the possibility of relating the development of learning and retention, and in the present case also persistence. to the maturation of certain structures or systems (Altman, Brunner, \& Bayer, 1973; Douglas, Peterson, \& Douglas, 1973). Such experimentation also provides an opportunity to examine the plausibility of employing the same hypothetical mechanisms to account for a variety of behavioral phenomena. For example, since it is hypothesized that phenomena like the frustration effect (FE) and the partial reinforcement acquisition and extinction effects (PRAE, PREE) are related to mechanisms of primary $\left(R_{F}\right)$ and conditioned $\left(r_{F}\right)$ frustration (Amsel, 1958), the occurrence or nonoccurrence of such effects in rats at various chronological ages should be correlated with the presence or absence of corresponding mechanisms, as inferred from physiological considerations or from other independent behavioral observations.

We recently presented preliminary evidence on the development of persistence in the rat (Chen \& Amsel, 1975) which showed that persistence (resistance to extinction) following both partial (PRF) or continuous (CRF) reward acquisition was inversely related to age: subjects whose training started before 25 days of age were clearly more persistent than those who were trained after 30 days of age. The latter group showed the typical adult pattern of extinction. At both ages, however, the PREE was obtained. As suggested by Chen and Amsel, improvements in experimental procedure, such 


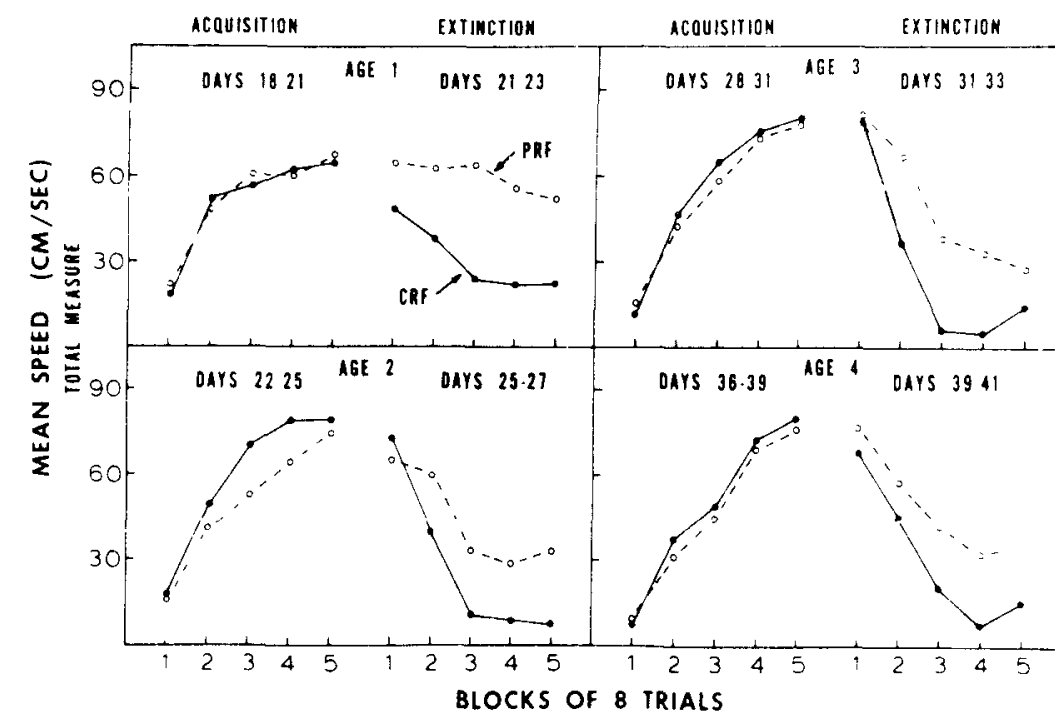

Figure 1. Acquisition and extinction curves for Experiment I, showing the PREE at each of four ages in terms of speeds for the total alley measure.

as confining training to a shorter period of time and including several age groups within the same experiment, would give a more accurate picture of how persistence develops in the rat.

We report three experiments in which acquisition and extinction of an instrumental appetitive response are employed to investigate persistence in the development of young rats of various ages. In these experiments, the acquisition (treatment) period is restricted to about 21/2 days in Experiments I and II, and to $4 \frac{1}{2}$ days in Experment III.

\section{EXPERIMENT I}

In the first experiment, we examined the effects of PRF and CRF treatments on acquisition and extinction in rats $18,22,28$, and 36 days of age at the beginning of training. It seemed important to determine whether rats differing in age by only a few days would show different patterns of persistence and whether we could find a transitional age at which differential persistence following PRF and CRF training (the PREE) is first revealed, perhaps within the 20- to 25-day range which seems important in other behavioral and physiological experiments in the rat.

\section{Method}

Subjects. These were 48 albino rats of the Holtzman strain bred in our laboratory. They came from litters culled within 3 days of birth to seven to nine pups. Beginning with weaning on Day 17 , the pups were assigned to one of four equal-sized groups randomly selected from the colony stock with the restriction that at least four litters were represented within a group and that an equal number of males and females were assigned to each group. They were then housed singly in standard laboratory cages with water continuously available. Purina Lab Chow was provided ad lib between weaning and the initial deprivation phase. The four groups formed were randomly assigned to four training-age conditions.

Apparatus. The straight alley was the same as described by Chen and Amsel (1975). Briefly. it consisted of a $27-\mathrm{cm}$ start- box, a $76-\mathrm{cm}$ runway, and a $30-\mathrm{cm}$ goalbox, all with internal dimensions of $7.6 \times 7.6 \mathrm{~cm}$ and covered with transparent Plexiglas. The startdoor was opaque Plexiglas. When raised, a microswitch mounted above the door started the first of three Standard Electric timers. Interruption of three photobeams positioned at consecutive $30.5-\mathrm{cm}$ distances from the startdoor yielded start, run, and goal times, respectively. An aluminum door separated the alley from the goalbox. The foodcup, located $12.5 \mathrm{~cm}$ beyond the last photobeam and mounted on the back wall of the goalbox, was a semicircular sheet metal trough extending the width of the goalbox. The lip of the cup was $2.5 \mathrm{~cm}$ above the floor and its depth was $12.5 \mathrm{~cm}$. Except where noted, the entire apparatus was painted flat black.

Procedure. The four groups of 12 rats each started training at 18 (Age 1), 22 (Age 2), 28 (Age 3), and 36 (Age 4) days of age.

Subjects were deprived of food for $24 \mathrm{~h}$ before the first session of acquisition tranning. Thereafter the amount of food provided daily, including amounts given as reward, was 6 g per day between Days 18 and $25,7 \mathrm{~g}$ between Days 26 to 30 , and $8 \mathrm{~g}$ between Days 30 and 42. Pilot work indicated that this deprivation schedule produces an apparently healthy yet hungry subject. Rats between 18 and 40 days of age given this same deprivation treatment showed a weight loss of $10 \%$ to $15 \%$ at the end of the first 24-h deprivation interval, followed by a daly gain in body weight of 1.5 to $2.5 \mathrm{~g}$,

Age groups were split equally into PRF and CRF conditions. The reward schedule for the PRF groups was as follows RNNR RNRN RRNN NRNR NRRN NNRR RNNR RNRN RRNN NRNR. Sessions occurred twice each day, separated by $12 \mathrm{~h}$. Subjects were run from wooden holding cages in two squads of 24 subjects ( 3 subjects per cell in each squad) and were returned to their home cages at the end of each session, where they were fed approximately 30 min later. Session 1 involved a single rewarded goalbox placement followed by two trials in the runway. The second session involved six trials, and each of the following sessions, eight trials. Goalbox confinement was approxımately 30 to $40 \mathrm{sec}$ on rewarded trials (time required to consume the pellets) and $25 \mathrm{sec}$ on nonrewarded trials and in extinction Intertral interval was 20 to $25 \mathrm{~min}$. Reward was two $45-\mathrm{mg}$ Noyes food pellets. There were 40 trials in acquisition and 40 in extinction.

\section{Results and Discussion}

Mean running speed over the entire alley $(\mathrm{cm} / \mathrm{sec})$ in blocks of eight trials is shown in Figure 1. This will be 
referred to as "total" speed. Variables included in the analyses of variance performed on the speed scores were two between-group variables, age and group (schedule of reward), and two within-group variables, blocks of trials and trials within blocks. Analyses are reported not only for the total speeds measure (start, run, and goal combined), but also for the separate measures wherever this provides additional information.

Acquisition. All groups showed significant improvement in runway performance characteristic of acquisition. There was a significant group effect for the total measure, indicating that $\mathrm{CRF}$ animals ran faster than PRF $[F(1 / 40)=6.50, p<.01]$, which was attributable mainiy to the difference in Age $2[F(1 / 10)=9.26$, $\mathrm{p}<.01]$. There was no significant Group by Block interaction at any age. Generally, in the seperate measures, PRF training tended to result in slower running than CRF training, particularly in older rats, and the classic partial reinforcement acquisition (PRAE) (Goodrich, 1959; Haggard, 1959), which we found in rats trained between 30 and 44 days of age (Chen \& Amsel, 1975, Experiment I), is not demonstrated in any of the present age groups. Of course, the conditions of the two experiments are very different not only in respect to age, but also for reward magnitude and number of trials per day. Terminal speeds in acquisition for Age 1 were lower than for Age 4, with Ages 2 and 3 reaching intermediatel levels, but the number of trials to reach near-asymptotic speeds was less for Age 1 than for the other three ages. These observations are supported statistically by a significant age effect $[F(3 / 40)=4.45, p<.01]$ and a significant Age by Block interaction $[\mathrm{F}(12 / 160)=5.16, \mathrm{p}<.001]$.

Extinction. The block effect was highly significant, reflecting decreasing speeds in extinction. Inspection of Figure 1 suggests a PREE, greater resistance to extinction following PRF than following CRF training, in all four age groups. However, since the extinction performances of the CRF groups reached their lowest level in the third block of trials and speeds increased or stayed the same in Blocks 4 and 5, only Blocks 1 to 3 (24 trials) were subjected to analysis. At Age 1 there was a significant group effect $[F(1 / 10)=33.97, p<.001]$ and Group by Block interaction $[F(2 / 20)=3.69, p<.05]$. At Age 2 there was a significant Group by Block interaction $[F(2 / 20)=4.63, p<.02]$, while at Age 3 the group effect $[F(1 / 10)=14.40, p<.01]$ and the Group by Block interaction $[F(2 / 20)=6.00, p<.01]$ were significant. At Age 4, the group effect was significant $[F(1 / 10)=7.71, \mathrm{p}<.01]$.

A comparison of extinction rates across Ages 1 to 4, following CRF and PRF acquisition combined, revealed a highly significant Age by Block interaction, $[F(6 / 80)=$ $5.10, \mathrm{p}<.001]$, indicating that extinction slopes steepen as age increases. Further, orthogonal comparisons showed that the absolute magnitude of the difference between the CRF and PRF groups, collapsed across blocks, was significantly greater in Age 1 than in the other three ages $[F(1 / 40)=27.08, p<.001]$. This is more clearly illustrated in Figure 2, where the extinction comparisons among ages are shown separately for the CRF and PRF groups. The left panel shows that the slope of the CRF extinction curve for Age 1 is much flatter, and terminal extinction speed higher, than in the three older groups, whose rates are indistinguishable from each other. This is supported statistically by a significant Age by Block interaction $[F(1 / 40)=2.76$, $\mathrm{p}<.02$ ] and, comparing Age 1 with the other three age groups, a significant group main effect $[\mathrm{F}(1 / 40)=12.45$, $\mathrm{p}<.001]$. No adjustment in the extinction scores was made to accommodate differences between Age 1 and Ages 2 to 4 in terminal acquisition, since such a conversion would only serve to magnify the differences under consideration and would not alter the main conclusions.

The comparison across age groups of extinction following PRF is presented in the right panel of Figure 2. The apparent differences in slope are confirmed by a significant Age by Block effect $[F(6 / 40)=2.98$, $p<.01]$. Orthogonal partitioning of the Age by Block interaction shows that Ages 1 and 2 differ significantly from Ages 3 and $4[F(1 / 40)=7.42, p<.01]$, and that
Figure 2. Terminal acquisition (TA) and extinction curves for the CRF and PRF conditions shown separately with age as the parameter in each case.

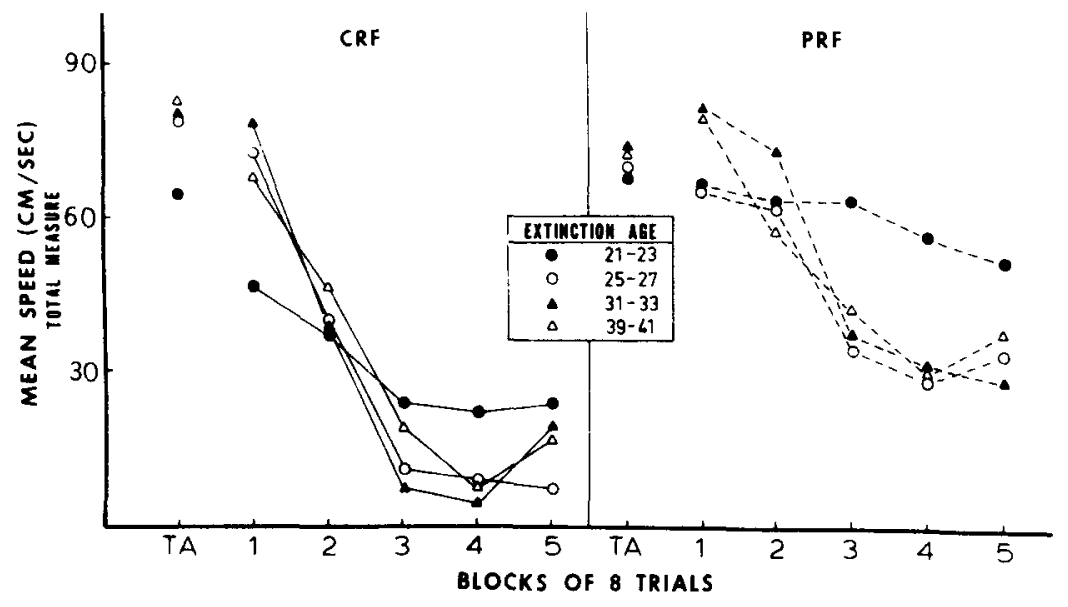




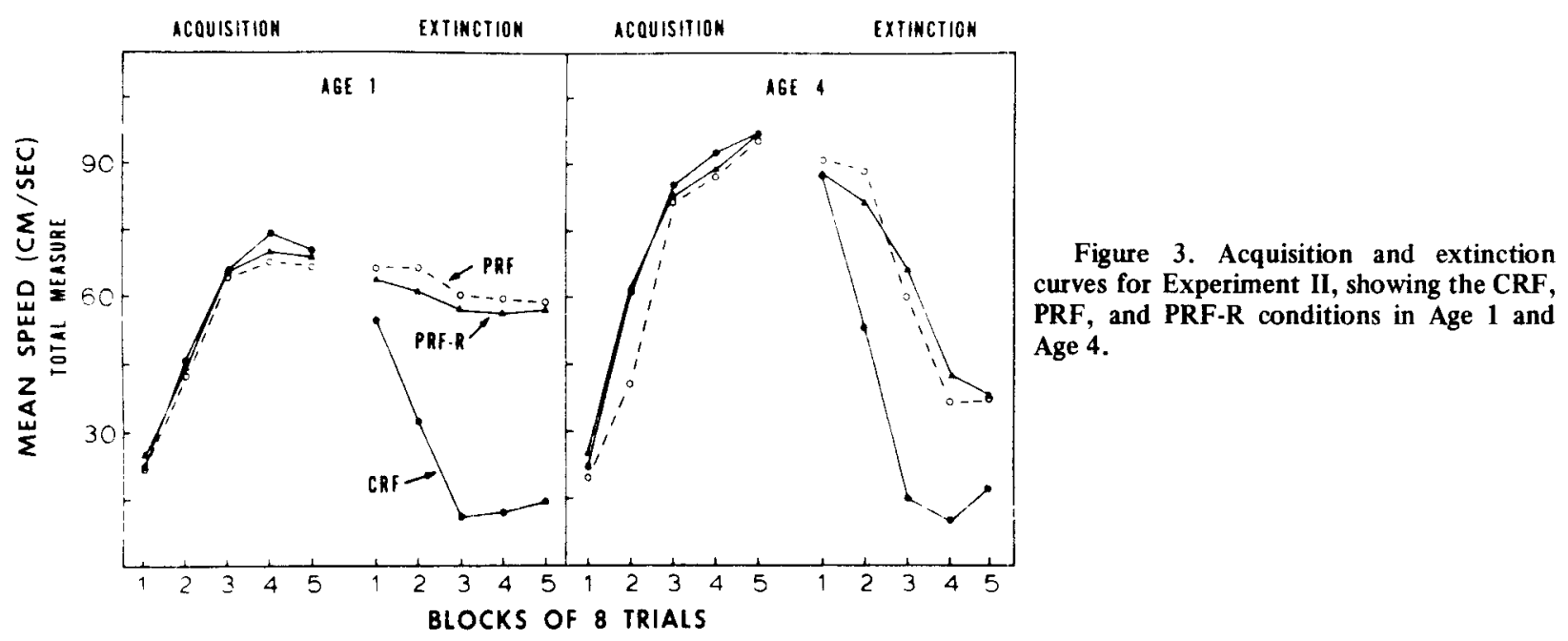

Age 1 differs from Age $2[F(1 / 40)=7.33, p<.01]$. This is interesting because, unlike the case following CRF, Age 1 and Age 2 combined differs from Ages 3 and 4 on the basis of slope in the initial three extinction sessions. In this regard, it is notable that the speeds of Ages 1 and 2 are the same in terminal PRF acquisition and in the first two blocks of extinction. Beyond that point, the youngest animals maintain strong persistence through Day 23, while the 25- to 27-day-olds show a sharp decline in speed on the 26th day.

The general picture in extinction is as follows: (a) Rate of extinction increases as a function of age, the main contribution to this effect being that, following both CRF and PRF training, the youngest animals (trained from Days 18-21) are significantly more resistant to extinction than their older counterparts. (b) While the PREE is found in all four age groups, the absolute magnitude of the effect is larger in Age 1 than at the other ages.

\section{EXPERIMENT II}

Although the general relationship of age and resistance to extinction in Experiment $I$ is not in disagreement with the hypothesis that weanling rats lack "inhibitory" mechanisms, a problem arises when this interpretation is applied to the PREE, which was not only present but quite substantial in the youngest age group. Most theoretical accounts of the PREE in adult rats implicate in the subsequent persistence some active feature of nonreward in PRF acquisition (e.g., Amsel, 1958; Capaldi, 1967). It is, of course, conceivable that active nonreward and/or frustrative mechanisms are not factors in the apparent PREE shown in our youngest animals, and Experiments II and III are addressed to this possibility. If it were the case that weanling rats, who are said to have an inhibitory deficit, are capable of learning only behaviors broadly classified as "appetitive-approach" or "excitatory," then the PREE at our Age 1 could be the result, not of the interspersed nonrewards in the PRF condition, but of the larger number of rewarded trials ( 40 vs. 20 ) in the CRF condition. In adult rats, it frequently has been shown, paradoxically, that beyond a given small number of trials, the larger the number of CRF trials the faster the subsequent extinction (see Sperling, 1965, for a review). Experiment II tested the possibility that in our youngest animals the PREE was in fact such an overlearning-extunction effect (OEE). This was accomplished by including, in addition to the basic CRF and PRF groups of Experiment $\mathrm{I}$, a second PRF group in which number of rewards rather than number of trials was equated with the CRF group. In addition to Age 1 , Age 4 was included in the design for purposes of comparison and to replicate the differences in persistence across ages which had been shown in Experıment I.

\section{Method}

Subjects. Fortyeight albino rats were selected, from litters born in our laboratory, along the Ines described in Experiment Apparatus and procedure. The apparatus was identical to that employed in Experiment $I$.

Two CRF and two PRI. groups (Ages 1 and 4) received treatment exactly as they did in Experiment I $(\mathrm{N}=8)$. At each age, a third group, PRF with rewarded trials equated with CRF (PRF-R), recelved, in addition to the pattern of reinforcement given Group PRF, two rewarded trials at the beginning and at the end of each session. These two new groups had a total of 40 rewarded and 20 nonrewarded trials at the end of acquisition. All other procedural detals were the sarne as in Experiment I.

\section{Results and Discussion}

Acquisition. Mean speed $(\mathrm{cm} / \mathrm{sec})$ for the total measure is shown in Figure 3.1 As a group, Age 1 subjects attained a lower acquisition asymptote than Age $4[F(1 / 42)=4.02, p<.05]$, and the two age groups had different rates of acquisition, as indicated by a highly significant Age by Block interaction [F(4/168) $=12.67, \mathrm{p}<.0001]$.

Data from the start, run, and goal measures over the last three blocks of acquisition trials were examined separately at each age for evidence of a Goodrich-Haggard effect. In Age 1, the group effect was significant in 
the start $[F(2 / 21)=5.37, p<.01]$ and goal $[F(2 / 21)=$ $9.91, \mathrm{p}<.0011$ measures, and the Group by Block interaction was reliable in the goal $[F(4 / 42)=3.50$, $\mathrm{p}<.011$. Pairwise comparison among the three groups indicated that the PRF and PRF-R groups ran faster than the CRF group in the start, ps $<.05$, and slower in the goal, ps $<.001$. The results for Age 4 were highly similar: the group effect was reliable in all three measures, ps $<.05$, and the PRF and PRF-R groups had slower speeds in the goal than the CRF group, ps $<.05$. The PRF-R, but not the PRF, group was faster than $\mathrm{CRF}$ in the run measure $[\mathrm{F}(1 / 21)=5.93, \mathrm{p}<.05]$. Thus, unlike Experiment $I$, there is some indication here of the PRAE in both age groups.

Extinction. Over blocks of extinction trials, Age 1 was generally more persistent than Age 4, as indicated by a significant Age by Block interaction $[F(4 / 168)=21.79$, $p<.001]$. Between-age analyses for each of the reinforcement conditions showed that in every comparison persistence was greater in Age 1 than in Age 4: for CRF groups, $F(4 / 56)=4.81, p<.01$; for PRF, $F(4 / 56)=9.74, p<.0001$; and for PRF-R, $F(4 / 56)=$ $4.83, \mathrm{p}<.01$.

Within each age, the group main effects and Group by Block interactions were highly significant (all ps $<.001$ ), indicating the presence of a PREE at both ages. The question of primary importance was whether, particularly in Age 1, PRF-R differed in rate of extinction from PRF. As Figure 3 shows, the two partial reward groups were virtually identical at all ages (pairwise comparisons, ps $>.10$ ), but were together more persistent than CRF in each case (ps $<.001)$. The size of the PREE (Age by Group interaction) was greater in Age 1 relative to Age 4 in the start, $p<.001$, and run, $p<.05$, measures, but not in the total, $p>.06$.

Summarizing the results of Experiment II, it is clear that the PREE found in weanling rats is not an OEE attributable to the larger number of rewards received in the CRF treatment in acquisition. Rather, the indication is that nonreward during PRF acquisition is the critical factor in building persistence even in rats 18 to 20 days of age. The goal measure acquisition data, which are generally the most sensitive reflection of the aversive effects of PRF training in adult rats, argue that the weanling rat has the ability to suppress ongoing behavior, at least in the sense that it approaches less vigorously a goalcup from which food is absent on some trials than one in which food is present on all trials, even when overall reward frequency is equated. In extinction, however, the rate of extinction at the youngest age is significantly lower than at other young ages in both PRF and CRF conditions, suggesting that the effects of nonreward in extinction at this age are relatively weak. At the same time, as the data from both Experiments I and II show, the weanling PREE is at least as large as the adult-like PREE of the older animals, and, furthermore, this is not due to some artifact such as terminal acquisition differences. On the basis of these findings, we are led to conclude, at least tentatively, that the behavior of weanling rats is in some manner and to some degree subject to the frustrative and aversive properties of nonreward.

\section{EXPERIMENT III}

Both Experiments I and II demonstrate the existence of age-related persistence effects in rats at the preweanling to juvenile range. In the experimental study of the ontogeny of learning, the factors of incentive (reward magnitude), level of motivation (hunger, thirst, pain), and their interactions are particularly troublesome because there is no obvious way to hold them equal across ages. Because age-related differences in learning or persistence may be simply a function of such between-age variation in nondevelopmental factors, it is important to manipulate the level of such factors and determine if the age effect is eliminated.

Experiment III examined the effect of reward magnitude on persistence following CRF acquisition in rats trained and extinguished between 17.23 (Age 1) and $36-42$ (Age 4) days of age. There is ample evidence that, in adult rats, rate of extinction is directly related to magnitude of reward in CRF acquisition (e.g., Roberts, 1969). If this well established effect of reward magnitude on rate of extinction operates in preweanling and juvenile rats as it does in adults, and if preweanling (Age 1) rats are more persistent than juvenile (Age 4) rats at both reward levels ( $45 \mathrm{mg}$ and $300 \mathrm{mg}$ ), this would increase our confidence (a) that the relationships between age and persistence shown in Experiment I have general ontogenic significance, and (b) that persistence in rats from about $18-20$ days to adulthood involves similar mechanisms.

\section{Method}

Subjects. Twenty-four albino rats were selected from our laboratory colony along the lines set forth in Experiment I, except for time of weaning which occurred on Day 16 (approximately $24 \mathrm{~h}$ earlier than in Experiments I and II).

Apparatus and procedure. The apparatus was identical to that employed in Experiments I and II.

Two CRF groups started training at Age 1 ( 17 days) and two CRF groups started at Age 4 (36 days). ${ }^{2}$ At each age, one group received a $45-\mathrm{mg}$ Noyes pellet on each trial in acquisition (Group 45) and the other a 300-mg pellet (Group 300). With half of the subjects receiving a rather large pellet in acquisition, it was expected that the longer time needed to eat would increase session length if the same number of trials per session were run as in Experiments I and II. Therefore, to equate overall session length with the two previous experiments, only 6 trials per session were conducted in Experiment III. The only other significant procedural changes involved the total number of sessions in acquisition, 9 instead of 5, again one in the morning and one in the evening, and consequently the number of acquisition trials, 42. The numbers of trials in Sessions 1 to 3 were 1,2 , and 3 , respectively, and there were 6 trials in each subsequent session. Acquisition training was completed for Age 1 at 21 days of age and for Age 4 at 40 days of age. Extinction consisted of five sessions of 6 trials each. 


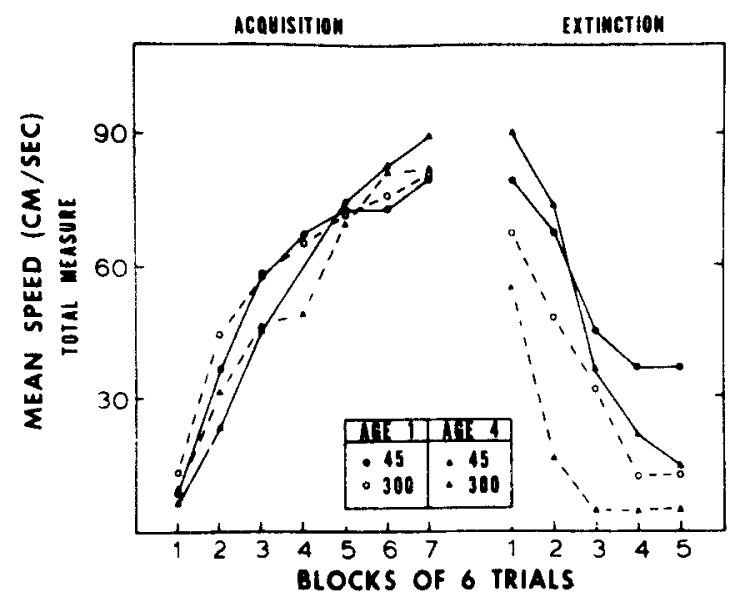

Figure 4. Reward magnitude effects (300 $\mathrm{mg}$ and $45 \mathrm{mg}$ ) in CRF acquisition and extinction for Ages 1 and 4 in Experiment III.

\section{Results and Discussion}

The between-groups variables included in the analyses were age ( 1 and 4 ) and reward magnitude (45 and 300), the within-groups variables, blocks of trials and trials within blocks.

Acquisition. The left-hand portion of Figure 4 shows the acquisition curves for the four groups for the total measure. There were no significant main effects of age or reward magnitude. Age 1 showed higher speeds than Age 4 over the first four blocks of trials, and there was a reverse of this difference in the last three blocks. This reversal from early to late acquisition is confirmed by a significant Age by Block interaction $[\mathrm{F}(6 / 120)=6.55$, $\mathrm{p}<.001]$. Similarly, the significant Reward by Block interaction $[F(6 / 120)=2.25, p<.05]$ reflects a higher level of performance of the $300-\mathrm{mg}$ groups early in acquisition and, for Age 4 but not Age 1, a crossover late in acquisition.

The triple interaction of age, reward, and block was not significant, which indicates that the effect of reward on rate of acquisition was the same at both ages, and that the effect of age on rate of acquisition was independent of reward magnitude. The evidence in Experiments I and II that subjects in Age I perform better in the early stages of acquisition than the older subjects was confirmed in this experiment. Reward magnitude also had a slightly facilitative effect on rate of acquisition early in training. This relatively small effect, restricted to the early portion of training, was shown at both ages and is at odds with the general findings using adult rats (e.g., Roberts, 1969; Wagner, 1961). However, there are reports, consistent with the present results, that reward magnitude affects rate but not asymptotic level of acquisition performance (Black, 1969; Campbell, Batsche, \& Batsche, 1972).

Extinction. The principal results of Experiment III are shown in the right-hand portion of Figure 4. Since the first extinction block already involves six trials. analysis of just the five extinction blocks would fail to take into account the change in performance between terminal acquisition and the initial session of extinction. We have therefore included in the analysis the last acquisition block as the reference point. Further, bec:use the Age 4 300-mg group in particular reaches extinction asymptote by the third block of trials, the interaction terms involving this group over all extinction blocks will be statistically spurious because the overall slope of this curve is relatively more flat than are those curves for groups that reach asymptote later in extinction. For that reason, one of our analyses of extinction included the final block of acquisition (TA) and the first three blocks of extinction. Analysis of the data from the five extinction blocks does not lead to substantially different conclusions.

The two ages extinguished at different rates, Age 1 extinguishing more slowly, Age by Block $[F(3 / 60)=$ $4.04, p<.01]$. The main effect of age was also highly significant, $p<.01$. Collapsed across age, there was a significant Reward by Block interaction, $[F(3 / 60)=$ $6.98, \mathrm{p}<.001]$, the small-reward groups extinguishing more slowly than the large-reward groups. The main effect of reward size was also highly signilicant, $p<.01$. At Age 4, the main effect of reward and the Reward by Block interactions were significant $[F(1 / 10)=24.21$, $\mathrm{p}<.001$, and $\mathrm{F}(3 / 30)=6.51, \mathrm{p}<.001$, respectively $]$. At Age 1, only the main effect of reward reached an acceptable level of significance $[F(1 / 10)=4.78$, $p<.05]$; the interaction of Age by Reward was not significant. The data and analyses indicate that, even at Age 1. larget reward leads to a lower overall extinction curve, but that rate of extinction of rats which started CRF training at 17 days is not affected by size of reward, at least over the first three extinction blocks. The relative size of the reward-magnitude effect is smaller at Age 1 compared to Age 4 when TA and five blocks of extinction trials are considered, as indicated by the Age by Group by Block interaction $[F(5 / 100)=$ $3.39, \mathrm{p}<.011$.

Comparison of the $45-\mathrm{mg}$ and $300-\mathrm{mg}$ groups between Age 1 and Age 4 indicated that, at each reward level, younger rats were more persistent than therr Age 4 counterparts. Further, the differences in extinction as a function of age were larger following acqusition with 300 -mg reward than with $45 \mathrm{mg}$. In the 300-mg comparison, the main effect of age and the Age by Block interaction were significant across terminal acquisition and Blocks 1 to 3 of extinction $[F(1 / 10)=17.64$, $\mathrm{p}<.001$, and $\mathrm{F}(3 / 30)=2.81, \mathrm{p}<.05$, respectively $]$. At $45 \mathrm{mg}$, Age 1 and Age 4 were not reliably different across the same four blocks of trials, but did show different rates of extinction when terminal acquisition and all five blocks of extinction trials were considered $[F(5 / 50)=3.52, p<.01]$. Including all five blocks of extinction in the $45 \cdot \mathrm{mg}$ analysis is legitimate because a "floor effect" was not apparent in either age group.

In analyzing the differences between Age 1 and Age 4 for the reward magnitude effect. differences among alley 
segments proved important. As Figure 5 shows, there was virtually no difference in rate of extinction between the two reward groups in Age 1 across the first three blocks of trials in the start and run measures, while in the goal the effect was apparent even in the first block. In contrast, the differences in Age 4 emerged much earlier in extinction in the start and run measures, although at this age as well the goal measure was the most sensitive in reflecting the reward effect. These observations are supported statistically; the reward main effect for Age 1 was significant only in the goal $[F(1 / 10)$ $=7.61, \mathrm{p}<.02$ ], while for Age 4 it was highly reliable in both the run and goal measures $[\mathrm{F}(1 / 10)=5.93$, $\mathrm{p}<.03 ; F(1 / 10)=19.18, p<.0011$, and fell just short of significance in the start, $p<.10$. There is, therefore, some indication that, with respect to the effect of reward magnitude on extinction, the position in the alley in which resistance to extinction is evaluated is another way to set Age 1 apart from Age 4. Specifically, as distance from the goal increases, the effect is reduced much more sharply in Age 1 than in Age 4.

\section{GENERAL DISCUSSION}

There are several clear empirical findings in these experiments in the ontogeny of persistence in rats. The results of Experiment I are consistent with the preliminary observations of Chen and Amsel (1975) in demonstrating that rate of extinction of an instrumental appetitive response immediately following PRF and CRF acquisition increases over the age range 21 to 41 days. First, there was greater immediate persistence, both after CRF and PRF training, but particularly after the latter, in the youngest rats. Secondly, all groups showed a clear PREE, and the effect was largest in Age 1. Third, as Experiment II shows, the PREE in Ages 1 and 4, and by inference at intervening ages, cannot be attributed to differences in the number of rewarded trials in acquisition. And finally, rate of extinction was directly related to reward magnitude in CRF acquisition only in the oldest animals, although the larger reward did depress the overall level of persistence in Age 1.

The first finding of Experiment I, by itself, is entirely compatible with existing hypotheses for learning and memory in weanling rats. For example, the inhibitiondeficit (Altman, Brunner, \& Bayer, 1973; Douglas, 1972) and memory (Campbell \& Spear, 1972) positions both would predict faster extinction as age increases. According to the inhibition-deficit position, the altricial rat in the initial 3 or 4 weeks of life lacks maturity in the central systems and structures necessary for inhibition of behavior. If extinction of instrumental responding is said to be controlled by inhibitory processes, it follows that rate of extinction should become greater as these systems and structures mature.

The memory-deficit interpretation (e.g., Campbell \& Spear, 1972), originally intended to apply primarily to the ontugeny of long.term memory, would maintain that the greater persistence in weanling rats is due to poorer retention, between and within extinction sessions, of the preceding nonrewarded trials. The extinction differences, both after CRF and PRF training, would reflect poorer retention by the young rat of the outcome of preceding extinction trials. However, the second finding of Experiment $I$ is not easily incorporated by a straightforward inhibition deficit or by a memory hypothesis and is of particular theoretical importance. It establishes that even in rats younger than 21 days of age the introduction of nonrewarded trials in acquisition is a factor that is active and effective in increasing persistence (or further reducing "inhibition") in extinction. A statement that weanling rats lack the ability to withhold or inhibit responding, or find it more difficult to retain information between and within sessions, does not seem to address itself to the existence of the robust PREE in rats of this age. Of course, neither hypothesis, in its present form, was designed to cover the case of appetitive persistence, and neither offers a satisfactory explanation of the present results.

The third finding (Experiment II) eliminates an interpretation of the PREE in preweanling rats that would not require the presence of active features of nonreward in PRF acquisition. According to such an explanation, the differences in extinction are the result of a greater reward expectancy in extinction in the CRF than in the PRF group when trials are equated in acquisition. We equated number of rewards in acquisition in the CRF and PRF-R groups while still retaining intermittency of reward in the latter, and found the PRF-R group as persistent as the regular PRF group, a finding which is basically the same in preweanling rats as in adults. Again, the nonrewarded trials in acquisition must be producing the effect.

The fourth finding (Experiment III) of faster extinction following larger magnitudes of reward under CRF training in both preweanling and juvenile rats is again similar in direction to the data from adult rats. Like the results of Experiment II, this finding offers no particular basis for adducing completely different factors in

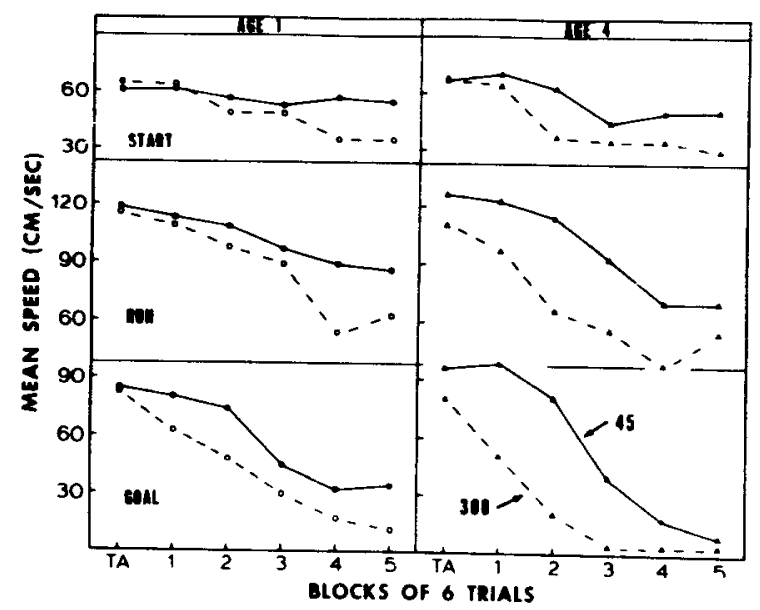

Figure 5. Alley-segment effects in terminal acquisition (TA) and extinction as a function of age and reward magnitude
following CRF acquisition. 
persistence in preweanling as compared to juvenile or adult rats. The same "paradoxical" effect of reward magnitude, but at a significantly smaller size at the youngest age, seems to be present at the two age levels investigated. One interpretation can be that at both age levels and in adults the larger magniude of reward in CRF acquisition results in a more intense reward expectancy which results in greater disruption (frustration. inhibition) of the ongoing response in extinction. Again it is difficult to reconcile this result with the inhibitiondefic1t and memory notions that have developed mainly out of the ontogenetic study of the acquisition and retention of certain fear-motivated responses.

These experiments point to the need for much more information. For example, we need to know whether there is long-term as well as short-term retention of the persistence established in PRF acquisition in the very young. In the experiments reported here and in the Chen and Amsel (1975) work, acquisition and extinction training covarted as a function of age. Consequently it is not possible to say that the differential extinction-persistence effects were attributable only to age in acquisition. Delaying the extinction test until all subjects reach maturity effectively 1solates the associations formed during acquisition from the influence of extinction factors that, early in life. may also be critically dependent on age. An experiment of this sort has recently been completed (Amsel \& Chen. in press). Further study in preweanling and even younger rats is required to evaluate one of the implications of Experiments II and III - that extinction in weanling and even younger rats may be dependent on factors not very different from those operating in adult persistence. Clearly, a well-defined set of experiments, directed at elucidating processes considered important from the standpoint of any theory of persistence (e.g., Ansel, 1972; Capald, 1967). might move us toward some reasonable hypotheses on the ontogeny of persistence. A potentially important byproduct of such programs of experimentation would be the discovery of rather precise behavioral indicants for the study of the functional significance of neural structures or processes, such as the hippocampus and cholinergic system, in the behavior of the developing organism.

\section{REFERENCES}

Altman, J., Brunner, R. L. \& Bayer, S. A. The hippocampus and behavioral maturation. Behavioral Biology, 1973, 8, 557-596. Altman, J.. \& Das, G. D. Autoradiographic and histological eviderice of postnatal hippocampal neurogenesis in rats. Journal of Comparative Neurology, 1965, 124, 319-336.

Amsel, A. The role of frustrative nonreward in noncontinuous reward situations. Psychological Bulletin, 1958,55, 102-119.

Amsel, A. Behavoral habituation, counterconditioning, and a general theory of persistence. In A. H. Black \& W. F. Prokasy (Eds.), Classical conditioning II. New York: Appleton-Century-Crofts, 1972 .
Amsel, A., \& Chen, J. S. Ontogeny of persistence: Immediate and long-term persistence in rats varying in training age between 17 and 65 days. Journal of Comparative and Physiological Psychology. in press.

Bitterman, M. E. Phyletic differences in learning. American Psychologist, 1965, 20, 396-410.

Black, $R$. W. Incentive motivation and the parameters of reward in instrumental conditioning. In W. J. Arnold and D. Levine (Eds.), Nebraska symposium on motivation. Vol. 17. Lincoln: University of Nebraska Press, 1969,85-137.

Bronstein, P. M., Neiman, H., Wolkoff, F. D., \& Levine, M. J. The development of habituation in the rat. Animal Learning \& Behavior, 1974, 2, 92-96.

Bronstein, P., \& S pear, N. E. Acquisition of a spatial discrimination by rats as a function of age. Journal of Comparative and $P$ hysiological Psychology, 1972, 78, 208-212.

Campbell, B. A. Developmental studies of learning and motivation in infraprimate mammals. In H. W. Stevenson, E. H. Hess. \& H. L. Rhelngold (Eds.), Early behavior Comparative and dovelopmental appraches, New York: Wiley, igōt.

Campbeil, B. A., Lytle, L. D., \& Fibiger, H. C. Ontogeny of adrenergic arousal and cholnergic inhibitory mechanisms in the rat. Science, $1969,166,635-636$.

Campbell, B. A., \& Jaynes, J. Effect of duration of reinstatement of retention of a visual discrimination learned in infancy. Developmental Psychology, 1969, 1, 71-74.

Campbell, B. A., Jaynes, J.. \& Misanin, J. R. Retention of a light-dark discrimination in rats of different ages. Journal of Comparative and Physiological Psvchology, 1968, 66, 467-472.

Campbell, B. A., \& Spear, N. E. Ontogeny of memory. Psychological Review, 1972,79, 215-236.

Campbell, P. E., Barsche, C. J., \& Batsche, G M. Spaced-traals reward magnitude effects in the rat Single versus multiple food pellets. Journal of Comparative and Physiological Psychology, 1972, 81, 360-364

Capaldi, E. J. A se'quential hypothesis of instrumental learning. In K. W. Spence and J. T. Spence (Eds.), The psychology of learning and motivation. Vol, 1 New York: Academic Press, 1967.

Chen, J.S., \& Amsel, A. Retention and durability of persistence acquired by young and infant rats. Journal of Comparative and Physiological Psychology, 1975, 89, 238-245.

Douglas, R. J. Pavlovian conditionng and the brain. In R. A. Boakes and $\mathrm{M}$ S. Halliday (Eds.), Inhibition and learning New York. Academic Press, 1972.

Douglas, R J., Peterson, J. J., \& Douglas, D.P. The ontogeny of a hippocampus-dependent response in two rodent species. Behavioral Blology, 1973, 8, 27-37.

Egger, G. J., Livesey, P. J., \& Dawson, R. G. Ontogenetic aspects of central cholinergic involvement in spontaneous alternation behavior. Developmental Psychobiology, 1973, 6, 289-299.

Goodrich. K. P Pfiformance in different segments of an instrumental response chain as a function of reinforcement schedule. Journal of Experimental Psychology, 1959, 57, 57-63.

Haggard, $D F$. Accussition of a simple runining response as a function of partial and continuous schedules of reinforcement. Psychological Record, 1959, 9, 11-18

Roberts, W. A. Resistance to extinction following partial and consistent reinforcement with varying magnitudes of reward. Journal of Comparative and Physiological Psychologv, 1969 $67,395-400$.

Sperling, S. E. Reversal learning and resistance to extinction: A review of the rat literature. Psychological Bulletin, 1965 . 63, $281-297$.

Wagner, A. R. Effects of amount and percentage of reinforcement and number of acquisition trials on conditioning and extinction. Journal of Experimental Psychology. 1961, $62,234-242$.

\section{NOTES}

1. Only the first eight tnals in each session of acquisition training for Group PRF-R were included in analyses. Examination of the four trials each session r.ot analyzed did not indicate different conclusions would have been reached if these extra trials were somehow accounted for.

2. These changes in procedure in Experiment III were introduced to equate with Experiments I and II the age (21-23 days) at which extinction performance was evaluated in Age 1.

(Received for publication July 10, 1975; revision accepted October $9+1975$. 